\title{
Four Dthats
}

\section{Stefano Predelli ${ }^{1}$}

Received: 23 December 2018 / Accepted: 15 May 2019 / Published online: 20 May 2019 (c) The Author(s) 2019

\begin{abstract}
The distinction between a merely 'rigidifying' dthat and a directly-referential take on dthat-terms is well known, and is explicitly highlighted by Kaplan in Afterthoughts, his 1989 commentary on Demonstratives. What is not equally widely recognized is that Afterthoughts also oscillates between three different directly referential proposals. This essay discusses the semantic and philosophical implications of these different directly-referential interpretations of 'dthat', paying particular attention to (a) the relationships between syntactic and propositional structure, (b) the structure and makeup of contexts in the semantics of indexical languages, (c) the significance of context shifting devices and of so-called operators on character, and (d) the aims and scope of propositional semantics.
\end{abstract}

Keywords Indexical $\cdot$ Semantics $\cdot$ Kaplan $\cdot$ Proposition

The distinction between a merely rigidifying 'dthat' and a directly-referential take on dthat-terms is well known and is explicitly highlighted by Kaplan in Afterthoughts (Kaplan 1989b), his 1989 commentary on his 1977 manuscript Demonstratives (Kaplan 1989a). What is not equally recognized is that Afterthoughts oscillates between at least three distinct directly-referential stances. This essay focuses on the relationships between the standard reading of directly-referential dthat-terms and two alternatives suggested by some less widely studied passages in Afterthoughts.

Why an entire paper on 'dthat'? For one thing, Kaplan's concoction has come to occupy a fundamental role in a variety of debates in semantics and philosophical logic. In its various guises, it has provided powerful tools for the analysis of diverse linguistic phenomena, including Kaplan's own target, the English demonstrative 'that', but also so-called complex demonstratives, definite descriptions, proper names, or kind-terms. ${ }^{1}$ Crucially, it has also played a central role in the development of a variety

\footnotetext{
1 For a small preliminary sample of various applications of 'dthat' see Almog (1981), Rey (1992), Kanterian (2009); see below for citations from the considerable literature on 'dthat' and demonstratives.

$凶 \quad$ Stefano Predelli

Stefano.predelli@nottingham.ac.uk

1 Department of Philosophy, University of Nottingham, Nottingham NG7 2RD, UK
} 
of meta-semantic views, having to do among other things with indexicality, with the role of different forms of context-dependence, and more generally with the aims and scope of propositional semantics. And so, unveiling the ideas lurking behind Kaplan's 'dthat' contributes to shed further light on these topics. Moreover, and perhaps most importantly, the discovery of hitherto ignored interpretations motivates novel formal devices, which are of independent interest from the viewpoints of philosophical logic and natural-language semantics.

Neither my methodology nor my aims, then, are exegetic. Although Kaplan's texts guide my discussion, my interpretations often rely on not always transparent (and occasionally superficially inconsistent) hints in Afterthoughts. I happily concede that my glosses make heavy weather of what may originally have been meant as casual suggestions, and that some of my results may well fail to be symptomatic of Kaplan's views. Still, the hints are there, and they deserve to be taken seriously.

Section 1 begins by rehearsing Kaplan's introduction of 'dthat' in Demonstratives, mostly with the aim of establishing the background for his later developments and amendments. Section 2 proceeds with what I call the standard reading of Kaplan's 1989 views on 'dthat', with particular attention to issues pertaining to the relationships between syntactic and propositional structure. Sections 3 and 4 flank this theme with related questions about the role and make-up of contexts, and unveil two different positions inspired by Afterthoughts.

\section{Beyond the first 'Dthat'}

In the formal framework of Demonstratives, expressions are assigned a 'denotation' (an extension) with respect to a context and what Kaplan calls a circumstance (Kaplan 1989a, p. 544). Abbreviating 'the extension of $e$ with respect to (or 'at') a context $c$ and a circumstance $w$ ' as $|e|_{\mathrm{c}, \mathrm{w}}$, Demonstratives introduces 'dthat' as follows,

(1) $I \mathrm{dthat}[$ the $F] \mathrm{I}_{\mathrm{c}, \mathrm{w}}=$ the unique individual $i$ such that $i=\mid$ the $\left.F\right|_{\mathrm{c}, \mathrm{c}_{\mathrm{w}}}$

where $c_{w}$ is the circumstance 'determined by' $c{ }^{2}$ Since 'dthat[the $F$ ]' is a term (Kaplan 1989a, p. 543), it follows from Kaplan's clause for atomic sentences (clause 2 in Kaplan 1989 a, p. 545) that, say, ' $G$ (dthat[the $F$ ]' is true at $c$ and $w$ iff $i \in|G|_{\mathrm{c}, w}$, with $i$ as in (1) (and with the obvious generalization to $n$-ary relation-symbols, $n>1$ ).

In the following section ('Remarks on the Formal System'), Kaplan introduces a familiar commentary to his double-indexed framework: the content $\{e\}_{c}$ of $e$ in $c$ is the function such that, for any $w,\{e\}_{c}(w)=|e|_{\mathrm{c}, \mathrm{w}}$. In particular,

(2) $\{\text { dthat }[\text { the } F]\}_{c}$ is the (constant) function $f$ such that, for all $w, f(w)$ is the unique $i$ such that $i=\mid$ the $\left.F\right|_{\mathrm{c}, \mathrm{c}_{\mathrm{w}}}$

\footnotetext{
${ }^{2}$ Kaplan (1989a, p. 546). I simplify considerably but harmlessly: (a) as hinted by my choice of ' $w$ ', I conceive of circumstances merely as possible-worlds, rather than as Kaplan-style world-time pairs; (b) I focus on the case of definite descriptions (as opposed to terms more generally) within the scope of 'dthat', (c) I take on board Kaplan's treatment of 'the $F$ ' as denoting an individual with respect to a context and a circumstance, and (d) I omit indications of models and assignments of values to variables. I also avoid mentioning the possibility that the associated description remains denotation-less, thus avoiding 'if any' caveats and bypassing a variety of niceties of little relevance for my aims.
} 
so that $\{G(\text { dthat }[\text { the } F])\}_{\mathrm{c}}$ is the function $f$ such that, for any $w, f(w)=$ Truth iff the aforementioned $i$ is in $\{G\}_{\mathrm{c}}(w)$. Accordingly, in Kaplan's formalism, what he calls 'contents' are functions from circumstances to extensions, that is, intensions.

The intensional definition of 'content' is apparently not a harmless arbitrary terminological decision, since Kaplan devotes considerable attention to it in the informal sections of Demonstratives: the representation of content as an intension, so we are told, 'is a handy one', even though 'one should note that contents which are distinct but equivalent are represented by the same intension' (Kaplan 1989a, p. 502). It would indeed be surprising if one did not note this consequence, given that, only a few pages before, Kaplan had vehemently stressed his dissatisfaction with that representation. There, we were explicitly invited not to 'think of propositions as sets of possible worlds, but rather as structured entities looking something like the sentences which express them' (Kaplan 1989a, p. 494, where, according to tradition, 'proposition' is a synonym for 'the content of a sentence'). The intensional understanding of content blatantly disregards this recommendation: for instance, for some simple term $t$, $\left\{G(\text { dthat }[\text { the } F]\}_{\mathrm{c}}\right.$ and $\{G(t)\}_{\mathrm{c}}$ may end up being the same function, notwithstanding the fact that these sentences, and hence presumably their structured contents, do not look that much alike.

Obliterating the presumed complexity and structure of content may well not be the end of the world, especially when it comes to results which may legitimately be indifferent to it. But what may be a 'handy' representation for a variety of Kaplan's purposes turns out to be particularly inappropriate when it comes to the study of a notion that is central in the informal parts of Demonstratives: the notion of direct reference. The idea of direct reference is indeed the protagonist of the paragraphs surrounding the aforementioned recommendation about how one should 'think of propositions', and is explicitly introduced with an allusion to propositional constituency: 'in the case of a singular term which is directly referential, the constituent of the proposition [expressed by a sentence in which it occurs] is just the object itself' (Kaplan 1989a, p. 494). Yet, the intensional disregard for structure inevitably yields a corresponding indifference for the peculiarities of direct reference. For instance,

(3) $\left\{G\right.$ (the $n\left[\left(\right.\right.$ snow is slight $\left.\wedge n^{2}=9\right) \vee\left(\sim\right.$ snow is slight $\left.\left.\left.\left.\wedge 2^{2}=n+1\right)\right]\right)\right\}_{\mathrm{c}}=$ $\{G(\text { three })\}_{\mathrm{c}}$

even though, in Kaplan's view, one proposition should contain 'a constituent which is complex', whereas the other should contain 'the object itself', as befits the directly referential status of 'three'. 3

Here as before, a nonchalant attitude may still not be out of place: although direct reference makes itself heard at the beginning of Demonstratives, it is perhaps legitimately left aside when it comes to those features of indexicality that may be uncovered from the intensional viewpoint. Yet, special care is anyway appropriate when it comes to at least one exemplar in Kaplan's toolbox, namely this essay's protagonist: 'dthat'. Indeed, Demonstratives does not simply gesture towards direct reference as an inde-

\footnotetext{
3 Or so I suppose for the example's sake (assuming that 'three' is an unstructured proper name, and that proper names are directly referential; modify my example if these assumptions turn out to be independently inadequate). The spectacularly syntactically complex exemplar on the left-hand side of (3) is Kaplan's (1989a, p. 494).
} 
pendently promising notion, which may nevertheless be put aside as we move on. Rather, it also explicitly invokes that idea in the very introduction to dthat-terms. The section entitled 'Dthat' begins thus: 'it would be useful to have a way of converting an arbitrary singular term into one which is directly referential' (Kaplan 1989a, p. 521). And it continues: 'why not ... introduce a special demonstrative which requires completion by a description and which is treated as a directly referential term ...? Why not? Why not indeed! I have done so, and I write it thus: dthat[ $\alpha$ ]' (Kaplan 1989a, p. 521).

The intensional framework, then, remains surprisingly indifferent to what, according to Kaplan's declared aims, seems to be the primary role of dthat-terms. Clearly, in parallel with the contrast in (3),

(4) $\left\{G\left(\right.\right.$ the $n$ [(snow is slight $\left.\wedge n^{2}=9\right) \vee\left(\sim\right.$ snow is slight $\left.\left.\left.\left.\wedge 2^{2}=n+1\right)\right]\right)\right\}_{\mathrm{c}}=$ $\{G($ dthat [the sum of two and one $])\}_{\mathrm{c}}$

even though, for Kaplan, one proposition should contain what he called 'a complex constituent', whereas the other should contain an individual.

What Demonstratives leaves unfinished is taken up with gusto in Afterthoughts, where propositional structure and direct reference come to the foreground. ${ }^{4}$ There, Kaplan's discussion begins with an explicit lamentation of the limits of his earlier formalism: 'the representation is possible world semantics temps us to confuse direct reference and obstinately rigid designation. Could anyone have confused them after the clear warnings of Sect. 4? Could I have? Yes. ... I find the confusion most evident in connection with dthat-terms' (Kaplan 1989b, p. 579).

The remainder of this essay is devoted to Kaplan's attempts at clearing that confusion, and at presenting an approach that properly recognizes the directly-referential nature of his concoction. Yet, as we shall see, the outcomes are not always as clear as one may have hoped, even though they are all instructive and worthy of consideration. In particular, I argue that at least three different devices emerge from Kaplan's informal comments on 'dthat': (a) the result of what, in Sect. 2, I call the standard reading of Afterthoughts, (b) the syntactically simple term I discuss in Sect. 3, and (c) the somewhat peculiar affair I introduce in Sect. 4.

Of course, all of these attempts continue to conform to the central constraints put forth in the intensional apparatus: if a dthat-term directly refers to $i$, it must still be the case that $i$ is selected as the unique individual who satisfies the description at the appropriate context. And so, the legacy of Demonstratives may concisely be summarized in terms of the following preliminaries to a directly-referential analysis of 'dthat':

(i) since what Kaplan called 'the propositional component' engendered by a directly-referential term is an object, if 'dthat' and/or dthat-terms are to follow Kaplan's intentions, their contents must be individuals. Moreover,

(ii) the choice of these individuals must depend on descriptive material: what is at issue with 'dthat' and/or dthat-terms are directly-referential terms 'whose referent is the denotation of the associated description' (Kaplan 1989a, p. 521), such as 'the $F$ ' in 'dthat[the $F$ ]'. Finally, as suggested by (1),

\footnotetext{
4 For classic developments of a Kaplan-inspired structured proposition framework see Salmon (1986) and Soames (1987); for more recent discussions see in particular King (2017).
} 
(iii) the denotation of 'dthat' and/or dthat-terms must be determined on the basis of the contextually privileged circumstances, as in $\mid$ the $\left.F\right|_{\mathrm{c}, \mathrm{c}_{\mathrm{w}}}$.

\section{Another 'Dthat'}

The relevant sections of Afterthoughts are headed by the question: 'are dthat-terms directly referential?' (Kaplan 1989b, p. 578, my italics). Kaplan's polemical tone against his former self hints at the following gloss: dthat-terms, though not formally recognized as directly referential, were 'originally intended' to be such. Much (though, crucially, not all) in the paragraphs that follow pursues this original intention.

If 'dthat [the $F$ ]' is directly referential, then, according to (i) above, its propositional contribution (its content) is an individual. Since contents are determined on the basis of character, it follows that, for any context $c$, $\{\operatorname{dthat}[$ the $F]\}(c) \in U$, where $\{e\}$ is the character of an expression $e$ and $U$ is 'the set of all individuals' (Kaplan 1989a, p. 543). ${ }^{5}$ Since, according to (ii), this individual is the 'denotation of the associated description', \{dthat [the $F]\}(c)$ must depend on the choice of 'the $F$ '. More precisely, given (iii), it is whatever is picked out by 'the $F$ ' at $c$ and at the circumstance it determines. So, in terms of character,

(5) $\{$ dthat $[$ the $F]\}=$ the function $f$ such that, for any $c, f(c)=\mid$ the $\left.F\right|_{\mathrm{c}, \mathrm{c}_{\mathrm{w}}}$

In the absence of a fuller picture of structured-proposition semantics, this assignment of character may well fail to provide the whole story about dthat-terms and the sentences in which they occur. In particular, (5) is, by itself, idle when it comes to propositional structure, for the obvious reason that it does not address the issue of the content of any sentential construct. A fortiori, then, it fails to provide an explicit indication of how the sentences in which dthat-terms occur may express propositions 'containing the object itself', as befits constructs involving a directly-referential term. Yet, at least for the present purposes, a familiar idea suffices to pave the way for a fuller treatment: at least in the simple case of a sentence $S$ of the form $G(t)$, the proposition expressed by $S$ at $c$ is the pair $<\{G\}_{\mathrm{c}},\{t\}_{\mathrm{c}}>$, that is, in the case of a term $t$ that is directly referential, a content that is true at $w$ iff the individual $\{t\}_{\mathrm{c}}$ is in the extension of $\{G\}_{\mathrm{c}}$ at $w$. For instance, the proposition expressed by the sentence on the right-hand side of (4) is the pair $<\{G\}(c)$, three $>$, unlike the more complex affair that is presumably appropriate for the syntactically cumbersome exemplar on the left-hand side.

And so, (5) provides a prima facie promising suggestion, which is at least in principle in the position of identifying the content of 'dthat[the $F$ ]' with an individual, and of associating the sentences in which it occurs with propositions containing an object as a constituent. Indeed, Kaplan seems to look at (5) with considerable sympathy: having insisted that a dthat-term's associated description is of 'semantic significance' but 'off the content record' (Kaplan 1989b, pp. 579-582), he concludes that 'the description completes the character of the associated occurrence of "dthat" (Kaplan 1989b, p. 581). Intriguingly, this idea had already been vented in a passage from Demonstratives devoted to demonstratives, that is, to the sort of expression which 'dthat'

\footnotetext{
5 That is, the content $\{e\}_{\mathrm{c}}$ of $e$ at $c$ is the result of applying $e$ 's character to $c:\{e\}(c)$.
} 
aims at formalizing: 'the character of a complete demonstrative [i.e., a demonstrative $d$ accompanied by a demonstration $\delta$ ] is given by the semantical rule' according to which it refers (at $c$ ) to whatever the accompanying demonstration picks out at $c$ (Kaplan 1989a, p. 527).

Unsurprisingly, then, (5) has become the standard reading of Kaplan's directlyreferential take on 'dthat'. ${ }^{6}$ Yet, Kaplan's supportive comments notwithstanding, it must not have been an easy position to live with. Indeed, as I am about to explain, some of its aspects blatantly clash with some central tenets in Kaplan's views about direct-reference, indexicality, and propositional structure.

As mentioned, Kaplan's original commitment to structured propositions rested satisfied with the idea that propositions look 'something like the sentences which express them' (Kaplan 1989a, p. 494). In turn, this presumably desirable match between syntactic and propositional make-up motivated his dissatisfaction with the intensional representation of content: as indicated in Sect. 1, the intension of a sentence is spectacularly indifferent to that sentence's structure. Yet, not any old structured content offered as a replacement for utterly unstructured intensions will do as a mirror of 'the sentence that expresses it'. Indeed, as I am about to explain, it is not at all clear how the proposal presented above induces propositional structures that appropriately reflect the structure of sentences containing occurrences of dthat-terms.

Given the recurring significance of the allegedly desirable match between propositional and syntactic structure, a snappy reminder of what is at issue is pedagogically desirable. It is a testimony to the continued interest in propositional semantics that an apt moniker has recently been proposed: according to what Bryan Pickel calls the Mirroring Thesis, the content of a sentence 'is a proposition composed of the [contents] of the sentence's constituents arranged in a structure which mirrors the structure of the sentence' (Pickel forthcoming). Or else, more generally: the content of a syntactically complex expression $e$ is composed of the contents of $e$ 's constituents, arranged in correspondence with $e$ 's syntactic structure.

The idea that the content of, say, ' $G$ (dthat[the $F]$ )' ends up being of the form $<\{G\}_{\mathrm{c}}, i>$, with $i$ an individual, may well satisfy the Mirroring Thesis to a greater extent than its representation as an unstructured intension. Yet, Kaplan's mirror here is very opaque indeed. For instance, the content of ' $G$ (dthat[the sum of two and one])' may well be aptly distinguishable from the content of the syntactically extravagant exemplar on the left-hand side of (3). But, continuing with my informal example, it is not at all different from the content of, say, ' $G$ (three)', notwithstanding the equally obvious structural differences between this sentence and ' $G$ (dthat[the sum of two and one])'. In general; as long as a dthat-term is understood as the combination of a definite description with a device for 'converting [it] into [a term] which is directly referential' (Kaplan 1989a, p. 521), it follows from the Mirroring Thesis that what ensues must be a correspondingly complex content. And, whatever that complex content may turn out

\footnotetext{
6 As a result, it has also become the standard reading of Kaplan's views on demonstratives. And so, according to Nathan Salmon, demonstratives, namely the expressions allegedly regimented by 'dthat', are 'uniformly given with a bracketed specification' in Demonstratives (Salmon 2002, p. 507). And, for Scott Soames, a term consisting of 'dthat' flanked by a description 'replaces the occurrences of [demonstratives] supplemented by [demonstrations]' (Soames 2012, p. 103).
} 
to be, it may surely not be the simple individual appropriate for a directly referential term. $^{7}$

There are no explicit indications that the concern for the Mirroring Thesis bears the responsibility for Kaplan's occasional allusions towards different directly-referential interpretations of 'dthat'. Yet, once again, a few textual hints are intriguing. More importantly, regardless of Kaplan's original intentions, the theoretical alleys they indicate are of independent semantic and philosophical interest. I thus turn to a different understanding of 'dthat' in the following section.

\section{A third 'Dthat'}

The following passage in Afterthoughts partly echoes the by now familiar complaint against the treatment of 'dthat' in Demonstratives:

on one interpretation, 'dthat' is a directly referential singular term and the content of the associated description is no part of the content of the dthat-term. On another interpretation, 'dthat' is syntactically an operator that requires syntactical completion by a description in order to form a singular term (Kaplan 1989b, p. 579).

The reason why this passage only partly reflects the considerations from Sect. 2 has to do with its emphasis on the 'syntactical completion' demanded by the definition of 'dthat' in the formalism of Demonstratives. Since that position is contrasted with the 'originally intended' view, it is reasonable to suppose that Kaplan's preferred directlyreferential take envisions a kind of 'dthat' for which such completion is not at issue. This interpretation is confirmed later on:

on [the intended] interpretation 'dthat' is a syntactically complete singular term that requires no syntactical completion by an operand (Kaplan 1989b, pp. 580-581).

This is a surprisingly bold conclusion, which, for one thing, demands a rephrasing of what Kaplan had written a few paragraphs before, in the passage I have cited at the beginning of this section: now, the point is not that 'the content of the associated description is no part of the content of the dthat-term', but rather that dthat-terms and their 'associated descriptions' must be left out of the picture altogether. Its boldness notwithstanding, this remedy to the alleged defects of Demonstratives must anyway

\footnotetext{
7 Interestingly, this problem reverberates at the level of character, even though it may be obscured by Kaplan's vague suggestion that 'dthat', being accompanied by a description that 'completes its character', must be endowed with an incomplete character (Kaplan 1989b, p. 581). After all, if incomplete characters are not characters, 'dthat' remains deprived of any semantic property recognizable in Kaplan's semantics (as explicitly lamented in Salmon 2002, p. 512 and Soames 2012, p. 104). But if 'dthat' has an (albeit incomplete or somehow special) character $k$ then, given that $\{\mathrm{dthat}[$ the $F]\}$ must be other than $k$, the role of 'the $F$ ' may seem to be that of a modifier $k$. And affairs of this sort seem to come perilously close to those 'attempts to operate on character' which Demonstratives deems to be 'impossible' (Kaplan 1989a, p. 510). (This issue is admittedly more complex than these brief remarks indicate, but a fully-fledged discussion of Kaplan-style monstrosity goes beyond the remit of this paper. For philosophical discussions of monstrosity and/or propositional semantics see for instance Rabern (2013) and Rabern and Ball (2017).
} 
have been unavoidable from the viewpoint of the Mirroring Thesis: as long as what is at issue is a complex expression such as 'dthat [the $F$ ]', what must ensue from any serious commitment to Mirroring is a content that may not be identified with a simple individual.

And yet, at least in this undeveloped form, the idea is also problematic: if 'accompanying descriptions' are expelled from syntax, dthat-terms disappear without being replaced by a clearly (or even not so clearly) defined simple term. One short passage hints at Kaplan's response:

'dthat' is no more an operator than is 'I', though neither has a referent unless semantically 'completed' by a context in the one case and a demonstration in the other (Kaplan 1989b, p. 581).

The analogy with 'I' is illuminating. For Kaplan (and presumably everybody else), 'I' is 'syntactically complete', and is endowed with a fully-fledged character $\{\mathrm{I}\}$, namely the function $f$ such that $f(c)=c_{a}$, the speaker (or 'agent') of $c$. The 'completion' required by 'I' is rather semantic: content is assigned to ' $\mathrm{I}$ ' only with respect to a context. If the analogy with 'dthat' is to be taken seriously, 'dthat' must follow a similar pattern of interpretive relativization. And so, a syntactically complete 'dthat' is 'semantically incomplete', because its referent can be identified only with respect to a suitable contextual parameter.

This much invites a different gloss on the idea that 'the description completes the character' of the associated occurrence of 'dthat'. The sense of 'completion' now at issue is the colloquial sense in which a function is completed by an argument: just as $\{\mathrm{I}\}$ is completed by $c$ so as to yield $c_{a}$, \{dthat $\}$ is completed by 'the $F$ ' in order to identify an individual. The following adjustments of Kaplan's original terminology suffice for a more precise presentation of this idea.

According to Demonstratives, contexts are quadruples including an individual, a location, a time, and a possible world. Characters are then defined as functions from contexts of this sort (Kaplan Contexts) to contents. The view entertained above demands a modification of this format: expressions are now evaluated not only with respect to a Kaplan Context, but also with respect to a description. This is so because 'dthat' makes non-trivial appeal to it: given any $c$ and 'the $F$ ', 'dthat' refers to whatever 'the $F$ ' denotes at $c$ and $c_{w}$. Accordingly, let us define an Extended Context as a quintuple of the form

(6) $c=<c_{a}, c_{l}, c_{t}, c_{w}, c_{d}>$,

with $c_{d}$ a definite description. ${ }^{8}$ Given a parallel adjustment of character as a function from Extended Contexts to contents, the character for 'dthat' is

(7) $\{$ dthat $\}=$ the $f$ such that, for any $c, f(c)=\left|c_{d}\right|_{c, c_{w}}$

This is a spectacular departure not only from the formalism of Demonstratives (my first 'dthat'), but also from the directly-referential take from Sect. 2 (the standard

\footnotetext{
8 Or, if you prefer, let us substitute the traditional idea of the content of an expression at a context with the notion of an expression's content with respect to an expression and a description (see Braun 1994, 1996 for discussions related to demonstratives). A more substantial alternative to the proposal in the main text includes as additional contextual parameters the content of the descriptions, rather than the description themselves. Much of what I am about to write may be adapted to this implementation, with opportune modifications.
} 
reading of Afterthoughts, i.e., my second 'dthat'). According to the view from the foregoing paragraph, dthat-terms are simply not allowed, and the descriptive material that previously accompanied 'dthat' is categorized as an element of a (somewhat unusual) contextual format. The general desiderata for a directly referential 'dthat' remain nevertheless in place: (i) the content of 'dthat', namely $\{d t h a t\}(c)$, is an individual, (ii) such an individual is 'the denotation of the associated description', now understood as a parameter $c_{d}$, where (iii) this description is evaluated with respect to the circumstances determined by $c$.

Admittedly, these desiderata remain in place at a cost: Extended Contexts are rather unusual affairs. They are, at the very least, structures that demand a few words of explanation. As I am about to explain, the issues that need to be addressed pertain to the central features of Kaplan's (1989a) apparatus. Unsurprisingly, they have to do with the nature and structure of contexts in the semantics for indexical languages.

The philosophical issue is somewhat complex, since Kaplan hints at different interpretations of the formal idea of a context, and since his contrasting attitudes have been taken on board by alternative developments of his semantics. Be that as it may, the admittance of expressions as elements of context, as with $c_{d}$ in my Extended Contexts, is surely a noteworthy departure from a standard and natural understanding of contextual parameters, namely as the extra-linguistic items required by the indexicals in the language. In particular, according to this widespread understanding, contexts are representative of concrete occasions of utterance: contexts 'represent situations in which an utterance might take place' (Rabern and Ball 2017, p. 1, summarizing Stalnaker 2014), and indexicals are evaluated 'with respect to ... the context of the actual speech act' (Schlenker 2003, p. 36). And so, in contrast to the format of Extended Contexts, contexts may well involve a speaker, a time, or a place, but not (or at least not obviously) words.

For the record, I am not sure whether Kaplan would unqualifiedly sympathize with this concern. In particular, at least in some parts of Demonstratives, he explicitly distances his semantics from the study of utterances and of the situations for their occurrences: 'it is important to distinguish an utterance from a sentence-in-a-context', he repeatedly warns (Kaplan 1989a, pp. 522 and 546). Even his terminology is occasionally designed to pursue a notion of context somewhat detached from the nitty-gritty of speaking: for instance, the individual occupying the position of $c_{a}$ in a context $c$ is often described as an 'agent' rather than a 'speaker'. And so, once this somewhat abstract idea of 'context' is taken on board, the notion of unusual contextual parameters, such as the descriptions in my Extended Contexts, may perhaps be less difficult to swallow.

Perhaps. ${ }^{9}$ As mentioned, the textual support for my third 'dthat' is limited. What receives a marginally more expansive discussion in Afterthoughts is yet a different approach to 'dthat'. Crucially, it is an approach which, perhaps independently of Kaplan's own intentions, paves the way towards independently important develop-

\footnotetext{
9 According to some commentators, Kaplan's own position on these issues is ambivalent: notwithstanding his distinction between sentences-in-context and utterances, he regiments contexts as $n$-tuples whose structure allegedly reflects that of (at least standard) occasions of speaking: contexts are inevitably proper, that is, they involve a speaker who is at the contextual location at the context's time (regarding propriety, see the considerable debate on apparently true occurrences of 'I am not here now', for instance Egan 2009; Mount 2008; Predelli 2005; and Stevens 2009).
} 
ments of (or amendments to) the standard approach to structured propositions. I turn to this fourth take on 'dthat' in the next section. ${ }^{10}$

\section{Towards a fourth 'Dthat'}

Sections 2 and 3 highlighted Kaplan's struggle with the Mirroring Thesis. Serious consideration for that thesis lead a few passages in Afterthoughts towards the idea of a syntactically simple 'dthat', implicitly accompanied by an unorthodox appeal to Extended Contexts. But pride of place was given to a suggestion that seemed content with the rejection of Mirroring, namely the view according to which syntactically complex dthat-terms have a simple individual as their contents.

It is presumably this ambivalent attitude towards Mirroring that leads Kaplan towards an underdeveloped (and, as far as I can tell, ignored within the subsequent literature) distinction of syntactic levels:

can an expression such as the description in a dthat-term appear in logical syntax but make no contribution to semantical form? ... there is no contradiction in such a language form (Kaplan 1989b, p. 582).

Kaplan's conclusion is prudent: though conceivable, this idea is also described as 'strange'. Yet, in a footnote, it is presented not only as consistent, but also as the formal counterpart of a natural language phenomenon:

we might think of the demonstration [that is, in the formal case, the description in a dthat-term] on the model of a term in apposition to the demonstrative [that is, 'dthat'] (Kaplan 1989b, p. 582, footnote 35).

This allusion to apposition remains unexplained. It seems nevertheless legitimate to suspect that Kaplan's analogy with what commonly goes under the name of 'apposition' must anyway be taken with an abundant grain of salt. What caught Kaplan's attention with run-of-the-mill appositives (such as non-restrictive relative clauses or nominal appositives) must surely have been their intuitively secondary discursive contribution, somewhat disentangled from the primary or official contents of their hosts (at least in some pre-theoretic vague sense of 'primary' and 'official'). ${ }^{11}$ Yet, when it comes to dthat-terms, Kaplan not only fails to even hint at any distinction between

\footnotetext{
10 This may be as good a place an any to mention an issue that I set aside in the main body of this essay, namely the proper analysis of multiple occurrences of 'dthat'. This issue is of particular urgency when it comes to my third 'dthat': if multiple occurrences of 'dthat' are admitted within a sentence, the required contextual resources arguably demand a sequence of descriptions, which ought to be appropriately related to each occurrence of 'dthat' (for instance, by appending numerical subscripts, as in 'dthat 1 '). It is further evidence of Kaplan's interest for the view put forth in this section that he explicitly struggles with this problem, at least when it comes to natural-language demonstratives: 'such cases seem to me to involve an exotic kind of ambiguity, perhaps unique to demonstratives' (Kaplan 1989a, b, p. 586; see also Braun 1996; Salmon 2002; Soames 2012; Gauker 2014; and Pickel et al. 2018).

11 In the influential framework developed in Potts (2003), these pre-theoretic ideas are systematically developed in a distinction between two types of content, so called 'at issue' and 'non-at-issue' contents; for discussions of Potts' distinction and of his framework see among many Ginzburg (1996), Amaral et al. (2007), and Simons et al. (2010).
} 
different levels of content, but also explicitly describes his project as that of excluding certain expression from anything that contributes to content in the first place. In a nutshell (and echoing Kaplan's 'off the record' metaphor, Kaplan 1989b, p. 579): natural-language appositives may perhaps remain off the main content record, whereas, according to the passage quoted above, the description in a dthat-term must remain entirely outside of it. $^{12}$

Leaving then aside Kaplan's interest in natural-language appositives, what must be explored is the very idea that an expression occurs as more than an idle embellishment (as must surely be appropriate for a part of logical syntax), and yet fail to contribute to content (as presumably befits anything that is not part of semantical form). Let then dthat-terms be terms in the language $L$ (of 'logical syntax') and envision a related language $S F_{L}$ of semantical form just like $L$, except for the exclusion of the dthat-term forming device 'dthat' and for the inclusion of a simple term 'Dthat'. The clause for the character of the $S F_{L}$-term 'Dthat' is

(8) $\{$ Dthat $\}=$ the function $f$ such that, for all $c, f(c)=c_{s}$

where $c_{s}$ is an element of what I call a Full Context, namely a quintuple $<c_{a}, c_{l}, c_{t}, c_{w}$, $c_{S}>\left(c_{s} \in \mathrm{U}\right)$. And as usual, continuing to focus on the simplest cases for conciseness' sake, the content at $c$ of an $S F_{L}$-sentence of the form $G(t)$ is the pair $<\{G\}_{\mathrm{c}},\{t\}_{\mathrm{c}}>$.

The assignment of contents to $L$-sentences is parasitic upon the results achieved by the semantics for $S F_{L}$. For instance, contents may be associated with $L$-sentences on the basis of the contents of their semantical forms, where an $S F_{L}$-sentence $S^{*}$ is the semantical form of an $L$-sentence $S$ iff $S^{*}$ differs from $S$ by substituting the occurrence (if any) of 'dthat [the $F]$ ' in $S$ with 'Dthat'. In particular, the content at $c$ of the sentence ' $G(\mathrm{dthat}[$ the $F])$ ' is the content of its semantical form at a distinct context $c^{*}$, as in

(9) $\{G(\text { Dthat })\}_{\mathrm{c}^{*}}$, with $c^{*}$ just like $c$ except that $c^{*}{ }_{s}=$ the unique $i$ such that $i \in$ $\{F\}_{\mathrm{c}}\left(c_{w}\right)$

This sketch unashamedly leaves aside a variety of obvious details and (possibly not so obvious) generalizations. The tediousness of a more appropriate formal presentation would anyway not be compensated by any spectacular semantic outcome: unsurprisingly, what ensues is that, in accordance with (i)-(iii) from Sect. 1, 'dthat[the $F$ ]' ends up contributing (at $c$ ) an individual chosen on the basis of the contribution of 'the $F$ ' at $c$ and $c_{w}$. Yet, as I indicate below, Kaplan's idea has interesting philosophical and meta-semantic repercussions, especially when it comes to the themes that have accompanied my discussion of his proposals: the role and structure of contexts, and the aims and scope of the Mirroring Thesis.

As for contexts, what plays the role of a contextual relatum in (8) is not the sort of affair which Kaplan took to be appropriate for $L D$ in Demonstratives, namely a

12 Indeed, in some views, the analogy between appositions (especially non-restrictive relative clauses) and Kaplan's new approach to dthat-terms may well also falter at the syntactic level: for Kaplan, the appositive description must occur within the 'logical syntax' of its host, whereas, for some, the role of a non-restrictive relative clause is that of a 'stylistic variants of coordinate sentences' (Quine 1960, p. 110), not unlike that of 'separate sentences' (Heim and Kratzer 1998, p. 88; for even more explicit commitments to a so-called radical orphanage approach to appositives see Fabb 1990; Haegeman 1988; and Burton-Roberts 1999; for a related position see McCawley 1988. For a contrary commitment to syntactic integration see for instance Potts 2003; Arnold 2007; Koev 2013; and Schlenker 2013). 
quadruple equipped only with the parameters required by what he calls 'pure indexicals' (in his system, 'I', 'here', 'now', and 'actually'). In this sense, Kaplan's fourth strategy echoes the suggestion that had already been vented in Sect. 3: 'dthat' and/or dthat-terms demand a dedicated contextual parameter, to no lesser extent than pure indexicals do. On the other hand, Full Contexts are not only different from the contexts of Demonstratives; they also differ from the Extended Context from Sect. 3, since what they demand is an individual, rather than a description. At least those who balked at the idea of a contextually relevant expression may find this a more palatable suggestion.

Moving on to the Mirroring Thesis: my interpretation of Kaplan's remarks on semantical form appealed to the distinction between (a) the syntactic layout generated by the lexicon and the formation rules of the original language $L$, and (b) a different, systematically related syntactic structure, the semantical form of the $L$-sentence under evaluation. This distinction is in turn responsible for an ambivalent attitude towards that other meta-semantic thread in my essay, the Mirroring Thesis. On the face of it, the outcome is a sort of compromise: although the make-up of a content does not mirror the structure of the $L$-sentence that express it, it accurately reflects the structure of a different syntactic affair, that $L$-sentence's semantical forms.

A compromise of this sort may perhaps be of some interest from the viewpoint of natural language semantics. Yet, in that case, the relationships between syntax and some other level of representation would surely need to be assessed on the basis of independent considerations. Surely, if semantical forms were constructed solely in order to supply the desired composition and structure of content, what would remain of the Mirroring Thesis would be an uninteresting triviality. ${ }^{13}$ As for Kaplan, the separation between logical syntax and semantical form may well remain the tentative outcome of a few passages written several years after Demonstratives. But it fittingly echoes the prudence with which the Mirroring Thesis had anyway originally been introduced: think of propositions 'as structured entities looking something like the sentences which express them', we had been told, with no further indication of how far that structural parallelism ought to go.

\section{Four Dthats: a final comparison}

Are the differences between my four proposals of a mere cosmetic nature? When it comes to the divide between a merely rigidifying 'dthat' and the alternative directly-referential proposals, Kaplan's own complaints in Afterthoughts suffice as a justification for a negative reply. Still, his casual shifts between the views discussed in Sects. 2-4 may suggest that an indifferent attitude is in order: all of the devices discussed above conform to the initial desiderata, namely (i)-(iii) from Sect. 1, and that may well be all that matters for a variety of semantic purposes.

Yet, as indicated above, the differences reverberate on a variety of foundational issues, such as the relationships between contexts and actual occasions of speaking, or

\footnotetext{
13 For a debate on the general relationships between syntactic and propositional structure see in particular Collins (2007), Cresswell (2002), King (2007), and Pickel (2017).
} 
the aims and scope of the Mirroring Thesis. Equally tellingly, they also exert importantly different pressures on the applications of Kaplan's frameworks to certain natural language phenomena. The debate on the most obvious of these applications, the case of demonstratives, nicely reflects Kaplan's oscillations among alternative approaches to 'dthat'.

For instance, modulo the syntactic representation of demonstrations as definite descriptions, Nathan Salmon develops the simple 'dthat' from Sect. 3 in the direction of a syntactically unaccompanied demonstrative, together with a contextual format that includes demonstrations: 'the demonstration does not belong in the expression. I say ... put the demonstration exactly where it has belonged all along: in the context' (Salmon 2002, p. 517). A different modification of Kaplan's (1989a) contexts follows the model provided by the Full Contexts from Sect. 4: according to the so-called Bare-Boned theory of demonstratives, their character is exhausted by their relation to an individual, the context's demonstratum. ${ }^{14}$ For others, finally, at least some demonstratives deserve a syntactically complex treatment inspired by the dthat-terms from Sect. 2: 'that man with the hat', for instance, is interpreted as a directly-referential affair, whose character is determined by the embedded 'man with the hat'. ${ }^{15}$ In a related contribution, similar ideas are developed by Josh Dever in an approach grounded on the idea of apposition, roughly in the spirit of the multi-dimensional syntax from Sect. 4 (Dever 2001). If the vivacious ensuing debates are anything to go by, a common commitment to directreference apparently leaves room for interestingly different implementations. ${ }^{16}$

Open Access This article is distributed under the terms of the Creative Commons Attribution 4.0 International License (http://creativecommons.org/licenses/by/4.0/), which permits unrestricted use, distribution, and reproduction in any medium, provided you give appropriate credit to the original author(s) and the source, provide a link to the Creative Commons license, and indicate if changes were made.

\section{References}

Almog, J. (1981). Dthis and Dthat: Indexicality goes beyond that. Philosophical Studies, 39, 347-381.

Amaral, P., Roberts, C., \& Smith, E. A. (2007). Review of the logic of conventional implicatures by Chris Potts. Linguistics and Philosophy, 30, 707-749.

Arnold, D. (2007). Non-restrictive relatives are not orphans. Journal of Linguistics, 43, 271-309.

Borg, E. (2000). Complex demonstratives. Philosophical Studies, 74, 193-219.

Braun, D. (1994). Structured characters and complex demonstratives. Philosophical Studies, 97, $229-249$.

Braun, D. (1996). Demonstratives and their linguistic meanings. Nous, 30, 145-173.

Braun, D. (2008). Complex demonstratives and their singular contents. Linguistics and Philosophy, 31, 57-99.

Burton-Roberts, N. (1999). Language, linear precedence and parentheticals. In P. Collins \& D. Lee (Eds.), The clause in English (pp. 33-51). Amsterdam: John Benjamins.

Caplan, B. (2003). Putting things in context. Philosophical Review, 112(2), 191-214.

Collins, J. (2007). Syntax, more or less. Mind, 116, 805-850.

Cresswell, M. J. (2002). Why propositions have no structure. Nous, 36, 643-662.

Dever, J. (2001). Complex demonstratives. Linguistics and Philosophy, 24, 271-330.

\footnotetext{
14 For developments see for instance Braun (1996), Caplan (2003), and Predelli (2012).

15 For discussions see for instance Braun (1994), Borg (2000), King and Stanley (2005), and Braun (2008).

16 I would like to thank the audience of the First CLE-SADAF Workshop on Philosophy of Language in Buenos Aires for their suggestions and feedback, and two anonymous referees for this journal for their constructive comments.
} 
Egan, A. (2009). Billboards, bombs and shotgun weddings. Synthese, 166, 251-279.

Fabb, N. (1990). The difference between English restrictive and non-restrictive relative clauses. Journal of Linguistics, 26, 57-78.

Gauker, C. (2014). How many bare demonstratives are there in English? Linguistics and Philosophy, 37, 291-314.

Ginzburg, J. (1996). Interrogatives: Questions, facts, and dialogue. In S. Lappin (Ed.), The handbook of contemporary semantic theory (pp. 385-422). Oxford: Blackwell.

Haegeman, L. (1988). Parenthetical adverbials: The radical orphanage approach. In S. Chiba, A. Ogawa, Y. Fuiwara, N. Yamada, O. Koma, \& T. Yagi (Eds.), Aspects of modern English linguistics (pp. 232-254). Tokyo: Kaitakushi.

Heim, I., \& Kratzer, A. (1998). Semantics in generative grammar. Oxford: Blackwell.

Kanterian, E. (2009). Puzzles about descriptive names. Linguistics and Philosophy, 32, 409-428.

Kaplan, D. (1989a). Demonstratives. In J. Almog, et al. (Eds.), Themes from Kaplan (pp. 481-563). Oxford: Oxford University Press.

Kaplan, D. (1989b). Afterthoughts. In J. Almog, et al. (Eds.), Themes from Kaplan (pp. 565-614). Oxford: Oxford University Press.

King, J. (2007). The nature and structure of content. Oxford: Oxford University Press.

King, J. (2017). Structured propositions. In E. Zalta (Ed.), The Stanford encyclopedia of philosophy. Stanford: The Metaphysics Research Lab.

King, J., \& Stanley, J. (2005). Semantics, pragmatics, and the role of semantic content. In Z. Szabo (Ed.), Semantics versus pragmatics (pp. 111-164). Oxford: Oxford University Press.

Koev, T. (2013). Apposition and the structure of discourse. Dissertation, Rutgers University.

McCawley, J. (1988). Parentheticals and discontinuous constituent structure. Linguistic Inquiry, 13, 91-106.

Mount, A. (2008). The impurity of 'pure' indexicals. Philosophical Studies, 138, 193-209.

Pickel, B. (2017). Structured propositions in a generative grammar. Mind, 128, 329-366.

Pickel, B., Rabern, B., \& Dever, J. (2018). Reviving the parameter revolution in semantics. In D. Ball \& B. Rabern (Eds.), The science of meaning: Essays on the metatheory of natural language semantics (pp. 138-171). Oxford: Oxford University Press.

Potts, C. (2003). The logic of conventional implicatures. Oxford: Oxford University Press.

Predelli, S. (2005). Meaning without truth. Oxford: Oxford University Press.

Predelli, S. (2012). Bare boned demonstratives. Journal of Philosophical Logic, 41(3), 547-562.

Quine, W. V. O. (1960). Word and object. Cambridge: MIT Press.

Rabern, B. (2013). Monsters in Kaplan's logic of demonstratives. Philosophical Studies, 164, 393-404.

Rabern, B., \& Ball, D. (2017). Monsters and the theoretical role of context. Philosophy and Phenomenological Research, 98, 392-416.

Rey, G. (1992). Semantic externalism and conceptual competence. Proceedings of the Aristotelian Society, 92, 315-333.

Salmon, N. (1986). Frege's puzzle. Atascadero: Ridgeview.

Salmon, N. (2002). Demonstrating and necessity. Philosophical Review, 111(4), 497-537.

Schlenker, P. (2003). A plea for monsters. Linguistics and Philosophy, 26, 29-120.

Schlenker, P. (2013). Supplements without bidimensionalism. Manuscript.

Simons, M., Tonhauser, J., Beaver, D., \& Roberts, C. (2010). What projects and why. Proceedings of SALT, 20, 309-327.

Soames, S. (1987). Direct reference, propositional attitudes, and semantic content. Philosophical Topics, 15, 47-87.

Soames, S. (2012). Philosophy of language. Princeton: Princeton University Press.

Stalnaker, R. (2014). Context. Oxford: Oxford University Press.

Stevens, G. (2009). Utterance at a distance. Philosophical Studies, 143, 213-221.

Publisher's Note Springer Nature remains neutral with regard to jurisdictional claims in published maps and institutional affiliations. 